\title{
Study Phase Transition Properties of a Ferroelectric Nanoparticle with the Usual Mean-Field Approximation
}

\author{
Yang Tong-Guang, Lu Zhao-Xin*, Shi Zhan \\ College of Mechanical Engineering \\ Linyi University \\ Linyi 276005, China \\ "e-mail: phylzx@gmail.com (L.Z.X)
}

\begin{abstract}
By using the usual mean-field approximation, the phase transition properties of a ferroelectric nanoparticle described by the transverse field Ising model have been studied. The exchange interactions and transverse fields at the surface are assumed to be different from those of the interactions parameters in the bulk. The phase diagrams for the ferroelectric nanoparticle with a size $S$ are calculated in the (Tc, Js) coordinates and the (Tc, $\Omega$ s) coordinates by two types of parameter modifications. In addition, the crossover features for interactions parameters of the ferroelectric nanoparticle are also examined.
\end{abstract}

Keywords-ferroelectric nanoparticle; transverse Ising model; phase diagram; Curie temperature

\section{INTRODUCTION}

In recent decades, the nano-materials have been paid attention more and more in experiment and theory [1-11]. In the experiment, it has been demonstrated that the magnetic properties of nanoparticle-based systems are deviated from the corresponding bulk nano-materials [1]. As we all know, these magnetic features can be easily changed due to the finite-size effects or surface effects while the particle size decreases. The study of nanoparticle magnetism seems to be one of the current topics in magnetism. In the theory, the transverse field Ising model has been thought to be a good candidate to describe the phase transitions in the ferroelectric nano-systems. The effective-field theory with correlations and the usual meanfield approximation have been used to inspect various physical properties of the ferroelectric nano-systems [3-11]. Kaneyoshi has studied the phase diagrams of a ferroelectric nanoparticle by changing the particle size $S$ as well as two ratios of $s$ and $p$ for the exchange interactions and the transverse fields between the corresponding value at the surface and the corresponding value in the bulk within the two frameworks of the effective-field theory with correlations and the usual mean-field approximation. The results indicate that the critical behaviors of interaction parameters are a little different from those of the ferroelectric thin films [3]. Afterwards, Kaneyoshi also calculated the thermal variations of the longitudinal and transverse magnetizations in the ferroelectric nanoparticle by the use of the usual mean-field approximation [4]. Recently, Kaneyoshi [5, 6] discussed the phase diagrams and magnetizations in the ferroelectric nanoparticles with different structures with the effective-field theory with correlations. Very recently, Lu [7] investigated the dependence of the phase diagrams on the exchange interactions and transverse fields of a ferroelectric nanoparticle in the $\left(T_{\mathrm{c}}, J_{\mathrm{s}}\right)$ coordinates and the $\left(T_{\mathrm{c}}, \Omega_{\mathrm{s}}\right)$ coordinates with the effective-field theory with correlations, however, this work has not been carried out by using the mean-field approximation up to now.

In this present paper, we will study the dependence of phase diagrams on exchange interactions and transverse fields for a ferroelectric nanoparticle in the $\left(T_{\mathrm{c}}, J_{\mathrm{s}}\right)$ coordinates and the $\left(T_{\mathrm{c}}, \Omega_{\mathrm{s}}\right)$ coordinates. The numerical results are compared with the corresponding results of the effective-field theory with correlations. Meanwhile, the crossover features for interactions parameters are also calculated.

\section{THE MODEL AND FORMULATIONS}

The ferroelectric nanoparticle with a certain size $S$ is assumed to be consisted of a two-dimensional arrangement of pseudo-spins, where the values of each pseudo-spin variable are $\sigma_{i z}= \pm 1$. Figure 1 shows the schematic illustration for the ferroelectric nanoparticle with a different size $S$. It can be found that the ferroelectric particle is consisted of the concentric hexagonal rings. The pseudo-spins on a ring $K(0<K \leq S)$ are on a same sublattice $K$. The pseudo-spins are connected to the nearest-neighbor pseudo-spins each other with spin exchange interaction. The Hamiltonian is $[3,4,7]$

$$
H=-\sum_{i j} J_{i j} \sigma_{i z} \sigma_{j z}-\Omega_{s} \sum_{i \in s} \sigma_{i x}-\Omega_{b} \sum_{i \in b} \sigma_{i x}
$$

where $\sigma_{i z}$ and $\sigma_{i x}$ are the pseudo-spin operators. $J_{i j}$ is the exchange interaction between the nearest-neighbor pseudospins at sites $i$ and $j . J_{i j}=J_{s}$ or $J_{i j}=J_{b}$ represents both pseudospins are at the surface ring or the others. $\Omega_{i j}=\Omega_{\mathrm{s}}$ or $\Omega_{i j}=\Omega_{\mathrm{b}}$ denotes the transverse fields when pseudo-spins are on the surface rings or in the bulk rings.

Under the usual mean-field approximation, the longitudinal magnetizations in the $z$-direction of the ferroelectric nanoparticle with $S=2$ are given as follows [3]

$$
\begin{gathered}
m_{1}=F_{b}\left(6 J_{b} m_{2}\right) \quad \text { фо } \rho \mathrm{K}=1, \\
m_{2}=F_{s}\left(2 J_{s} m_{2}+J_{b} m_{1}\right) \quad \text { фо } \rho \mathrm{K}=2,
\end{gathered}
$$




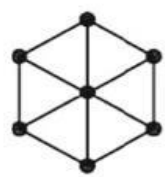

(a) $\mathrm{S}=2$

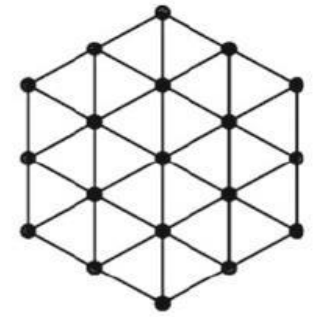

(b) $\mathrm{S}=3$

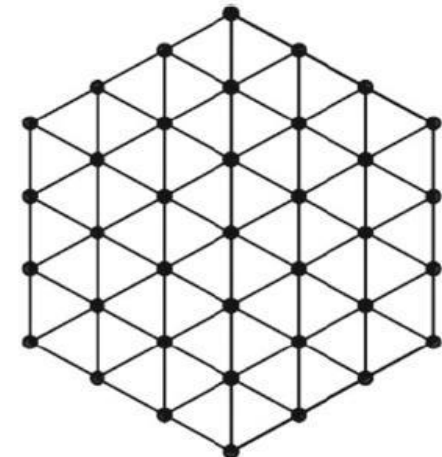

(c) $\mathrm{S}=4$

Figure 1. Schematic illustration of a ferroelectric nanoparticle with a size S.

where the functions $F_{\mathrm{b}}(x)$ and $F_{\mathrm{s}}(x)$ are defined as

$$
F_{b}(x)=\frac{x}{y_{b}} \tanh \left(\beta y_{b}\right)
$$

with

$$
y_{b}=\sqrt{x^{2}+\Omega_{b}^{2}},
$$

and

$$
F_{s}(x)=\frac{x}{y_{s}} \tanh \left(\beta y_{s}\right)
$$

with

$$
y_{s}=\sqrt{x^{2}+\Omega_{s}^{2}},
$$

$\beta=1 / k_{\mathrm{B}} T, \quad k_{\mathrm{B}}$ is the Boltzmann constant and $T$ is the absolute temperature.

Similar to the ferroelectric nanoparticle with $S=2$, the longitudinal magnetizations for the ferroelectric nanoparticle with $S=3$ can also be obtained [3]

$$
\begin{gathered}
m_{1}=F_{b}\left(6 J_{b} m_{2}\right) \quad \text { } о \rho \quad \mathrm{K}=1, \\
m_{2}=F_{b}\left(2 J_{b} m_{2}+J_{b} m_{1}+J_{b} m_{30}+2 J_{b} m_{31}\right) \quad \text { фо } \mathrm{K}=2,
\end{gathered}
$$

and

$$
\begin{array}{cc}
m_{30}=F_{s}\left(2 J_{s} m_{31}+J_{b} m_{2}\right) & \phi о \rho \mathrm{K}=3, \\
m_{31}=F_{s}\left(2 J_{s} m_{30}+2 J_{b} m_{2}\right) & \text { фо } \rho \mathrm{K}=3 .
\end{array}
$$

Thus, the coupled equations of magnetizations for the ferroelectric nanoparticle with a certain size $S$ can be easily derived with the framework of the mean-field approximation.

The right sides of the coupled equations for the ferroelectric nanoparticle with a certain size $S$ can be expanded. As a result, we can neglect the nonlinear terms and only take into account the linear terms. Thus, the transition temperature $T_{\mathrm{c}}$ can be derived as the functions of $J_{\mathrm{s}}, J_{\mathrm{b}}, \Omega_{\mathrm{s}}$ and $\Omega_{\mathrm{b}}$. The general equations for the two systems with $S=2$ and $S=3$ are obtained [3]:

$$
\begin{gathered}
1=6 A B_{1}+2 B_{2} \quad \text { фо } \rho \Sigma=2, \\
{[1-2 A(1+3 A)]\left[1-4 B_{2}{ }^{2}\right]=A B_{1}\left(5+8 B_{2}\right) \quad \text { фо } \Sigma=3,}
\end{gathered}
$$

with

$$
A=J F^{\prime}(0), B_{1}=J F_{s}^{\prime}(0), B_{2}=J_{s} F_{s}^{\prime}(0) .
$$

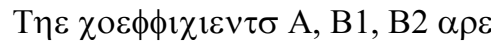

$$
\begin{gathered}
A=\left.\sinh \left(J_{b} \nabla\right) \cosh ^{5}\left(J_{b} \nabla\right) F_{b}(x)\right|_{x=0}, \\
B_{1}=\left.\sinh \left(J_{b} \nabla\right) \cosh ^{2}\left(J_{s} \nabla\right) F_{s}(x)\right|_{x=0}, \\
B_{2}=\left.\sinh \left(J_{s} \nabla\right) \cosh \left(J_{s} \nabla\right) \cosh \left(J_{b} \nabla\right) F_{s}(x)\right|_{x=0} .
\end{gathered}
$$

where $\nabla=\partial / \partial x$ is the differential operator and $e^{\alpha \nabla} f(x)=f(x+\alpha)$.

\section{NUMERICAL RESULTS}

In this section, we will dicuss the phase transition properties of a ferroelectric nanoparticle with a certain size $S$ from the general equations $[12,13]$. Usually, the phase diagram can be described by two ways: the relationships between the surface exchange interaction $J_{\mathrm{s}}$ and the Curie temperature $T_{\mathrm{c}}$ as well as the relationships between the
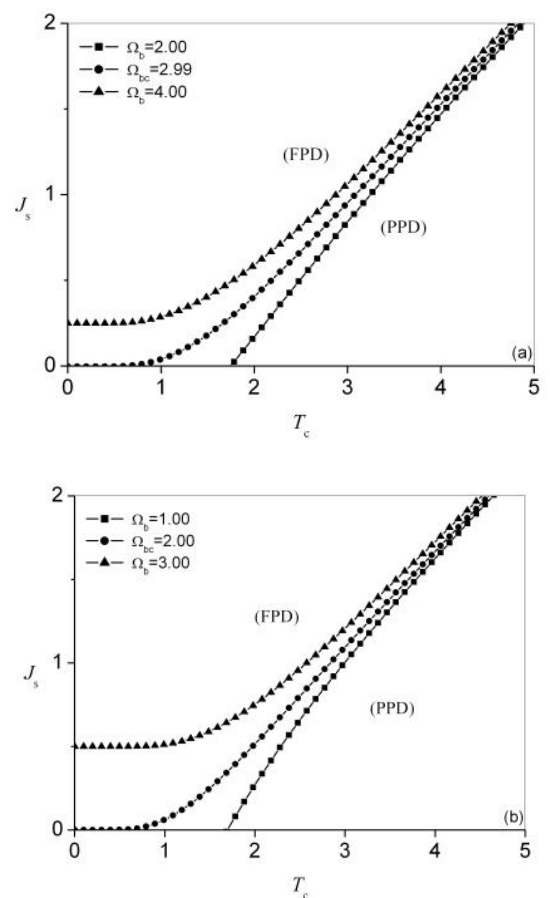

Figure 2. The dependence of the phase diagram on the bulk transverse field $\Omega_{\mathrm{b}}$. $J_{\mathrm{b}}=1.00, S=2$. (a) $\Omega \mathrm{s}=2.00$, (b) $\Omega \mathrm{s}=3.00$. 
surface transverse field $\Omega_{\mathrm{s}}$ and the Curie temperature $T_{\mathrm{c}}$, namely the curves of $J_{\mathrm{s}} \sim T_{\mathrm{c}}$ or $\Omega_{\mathrm{s}} \sim T_{\mathrm{c}}$. For simplicity and without loss of generality, we consider the ferroelectric nanoparticle with a particle size $S$ changing from 2 to 5 .

Figure 2 shows the dependence of the phase diagram on the bulk transverse field $\Omega_{\mathrm{b}}$, namely the curves of $J_{\mathrm{s}} \sim T_{\mathrm{c}}$ for different $\Omega_{\mathrm{b}}$. For a certain size $S$, it is obvious that the curve of $J_{\mathrm{s}} \sim T_{\mathrm{c}}$ depends sensitively on the bulk transverse field $\Omega_{\mathrm{b}}$. With the surface exchange interaction $J_{\mathrm{s}}$ increases, the Curie temperature $T_{\mathrm{c}}$ also increases monotonously. The smaller the bulk transverse field $\Omega_{\mathrm{b}}$, the larger the range of the ferroelectric phase region. The larger the bulk transverse field $\Omega_{\mathrm{b}}$, the larger the range of the paraelectric phase region. Thus, the phase diagram will be the ferroelectric-dominant phase diagram (FPD) or the paraelectric-dominant phase diagram (PPD). The FPD
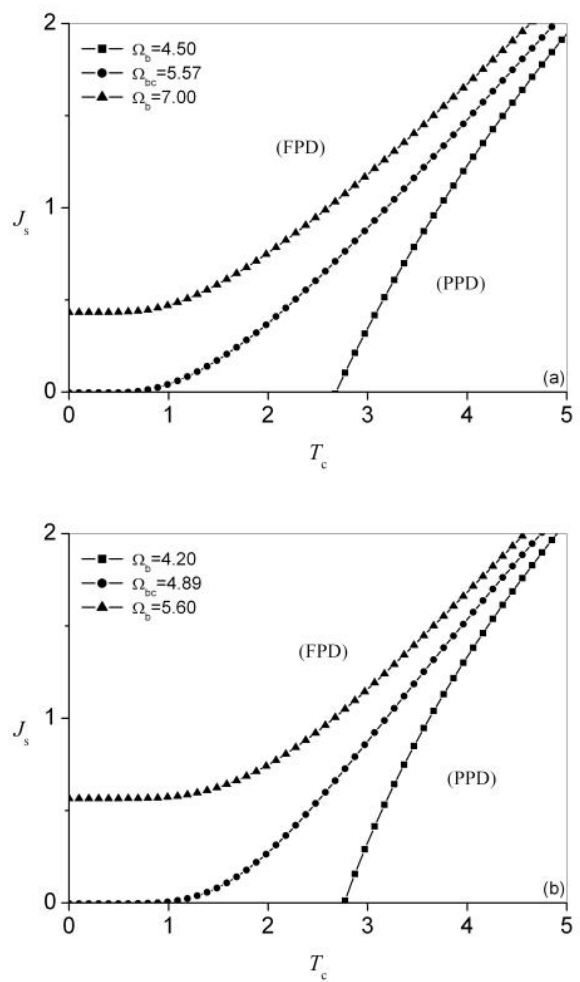

Figure 3. The dependence of the phase diagram on the bulk transverse field $\Omega_{\mathrm{b}}$. $J_{\mathrm{b}}=1.00, S=3$. (a) $\Omega \mathrm{s}=2.00$, (b) $\Omega \mathrm{s}=3.00$.

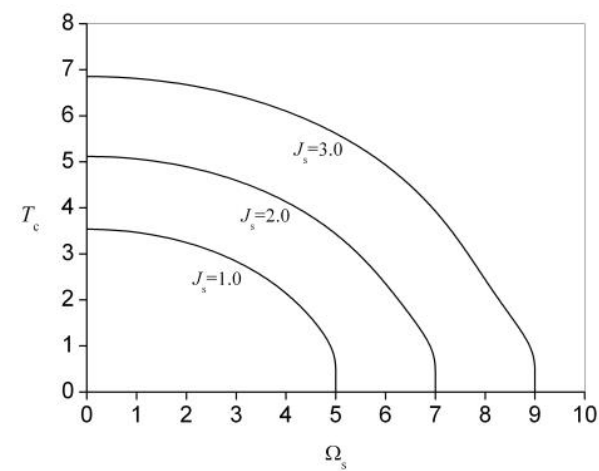

Figure 4. The dependence of the phase diagram on the surface exchange interaction $J_{\mathrm{s}} . J_{\mathrm{b}}=1.00, \Omega_{\mathrm{b}}=2.00, S=2$. denotes that the phase diagram is only in a ferroelectric phase state when the temperature is below a certain value without respect to the surface exchange interaction $J_{\mathrm{s}}$. That is to say, any $J_{\mathrm{s}}$ can result in a transition from the ferroelectric phase state to paraelectric phase state as the temperature increases. On the other hand, the PPD represents that the system is only in a paraelectric phase state when the surface exchange interaction $J_{\mathrm{s}}$ is below a certain value without respect to the temperature. In other words, only a larger $J_{\mathrm{s}}$ can result in a transition from ferroelectric phase state to paraelectric phase state as the temperature increases. Therefore, there exists a crossover value of the bulk transverse field $\Omega_{\mathrm{b}}$ while the phase diagram changes from the FPD state to PPD state. It is obvious that the crossover value $\Omega_{\mathrm{bc}}$ can also be obtained when the values of $J_{\mathrm{s}}$ and $T_{\mathrm{c}}$ reduce to zero at the same
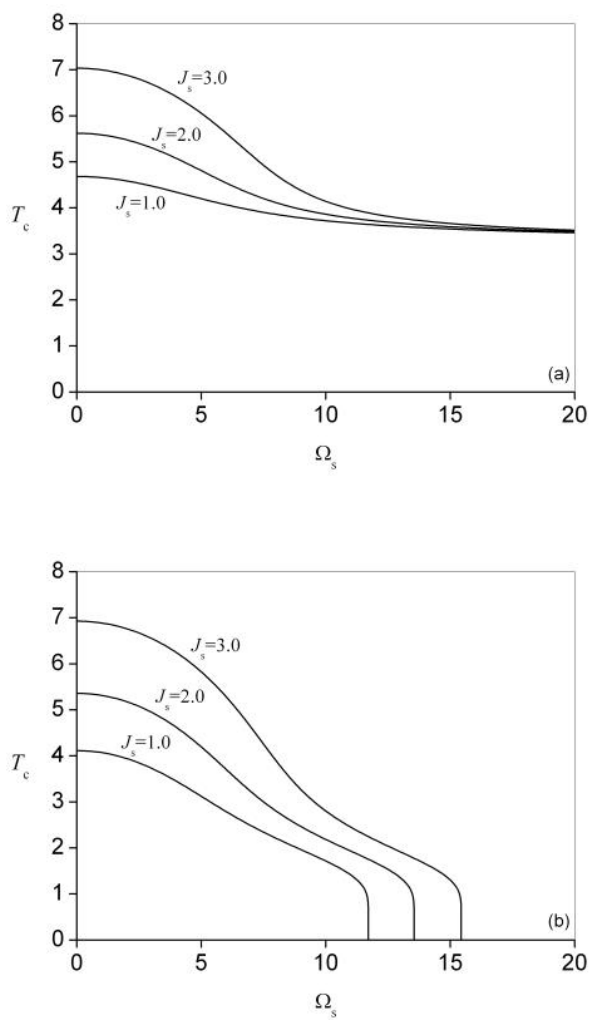

Figure 5. The dependence of the phase diagram on the surface exchange interaction $J_{\mathrm{S}} . J_{\mathrm{b}}=1.00, S=3$. (a) $\Omega_{\mathrm{b}}=2.00$, (b) $\Omega_{\mathrm{b}}=4.00$.

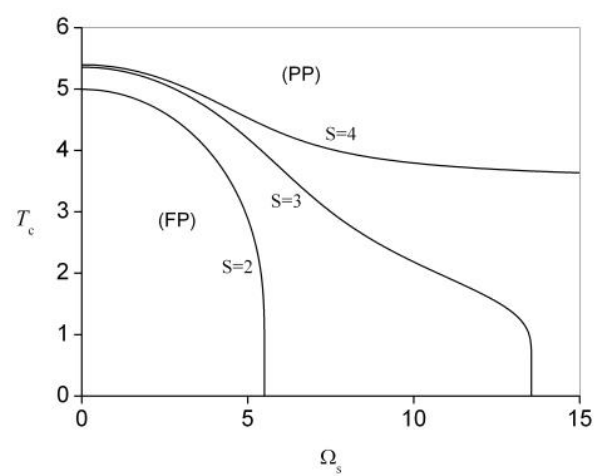

Figure 6. The dependence of the phase diagram on the ferroelectric nanoparticle size $S . J_{\mathrm{S}}=2.00, J_{\mathrm{b}}=1.00, \Omega_{\mathrm{b}}=4.00$. 
time. Meanwhile, the effect of the surface transverse field $\Omega_{\mathrm{s}}$ on the crossover value is also exhibited in Figure 2. The larger the surface transverse field $\Omega_{\mathrm{s}}$, the smaller the crossover value $\Omega_{\mathrm{bc}}$. Figure 3 is the same as Figure 2 but for a different particle size $S$. Obviously, the larger the particle size $S$, the larger the crossover value $\Omega_{\mathrm{bc}}$. These features in Figure 2 and 3 are similar to the corresponding result of the effective-field theory with correlations [5, 7].

Figure 4 shows the dependence of the phase diagram on the surface exchange interaction $J_{s}$, i.e., the curves of $T_{\mathrm{c}} \sim \Omega_{\mathrm{s}}$ for different values of $J_{\mathrm{s}}$. For a certain $J_{\mathrm{s}}$, with the surface transverse field $\Omega_{\mathrm{s}}$ increases, the Curie temperature $T_{\mathrm{c}}$ decreases monotonously. Finally, the Curie temperature $T_{\mathrm{c}}$ will reduce to zero at a critical value of the surface transverse field $\Omega_{\mathrm{s}}$. Meanwhile, the effect of the surface exchange interaction $J_{\mathrm{s}}$ on the curves of $T_{\mathrm{c}} \sim \Omega_{\mathrm{s}}$ can also be found in Figure 4. For a certain surface transverse field $\Omega_{\mathrm{s}}$, the larger the surface exchange interaction $J_{\mathrm{s}}$, the larger the Curie temperature $T_{\mathrm{c}}$. Furthermore, there is a critical value of the Curie temperature $T_{\mathrm{c}}$ when the surface transverse field $\Omega_{\mathrm{S}}$ reduces to zero.

Figure 5(a) is the same as Figure 4, but for a different particle size $S=3$. Now, it is obvious that the curves of $T_{\mathrm{c}} \sim \Omega_{\mathrm{s}}$ for different values of $J_{\mathrm{s}}$ will intersect at a common point as the increase of surface transverse field $\Omega_{\mathrm{s}}$. However, the curves of $T_{\mathrm{c}} \sim \Omega_{\mathrm{s}}$ will not intersect with the coordinate axis in $x$ direction irrespective of the value of surface transverse field $\Omega_{\mathrm{s}}$. Figure 5 (b) is the same as (a) but for a larger bulk transverse field $\Omega_{\mathrm{b}}$. Obviously, it is different from Figure 5(a), namely the curves of $T_{\mathrm{c}} \sim \Omega_{\mathrm{s}}$ for three different values of $J_{\mathrm{s}}$ intersect with the coordinate axis in $x$ direction. It indicates that the curves of $T_{\mathrm{c}} \sim \Omega_{\mathrm{s}}$ for different values of $J_{\mathrm{s}}$ depends sensitively on the bulk transverse field $\Omega_{\mathrm{b}}$.

Figure 6 shows the dependence of the phase diagram on the size $S$ of the ferroelectric nanoparticle. It can be seen from Figure 6 , the curves of $T_{\mathrm{c}} \sim \Omega_{\mathrm{s}}$ are plotted as the function of $S$. The two curves for $S=2$ and 3 intersect with the $x$ coordinate axis at a critical value of $\Omega_{\mathrm{s}}$, but the curve for $S=4$ does not intersect with the $x$ coordinate axis. These features are also similar to the corresponding result of the effective-field theory with correlations $[5,7]$.

\section{CONCLUSION}

In conclusion, the phase transition properties of a ferroelectric nanoparticle described by the transverse field Ising model have been studied by the usual mean-field approximation. By modifying exchange interactions and transverse fields at the surface, the phase diagrams in the $\left(T_{\mathrm{c}}, J_{\mathrm{s}}\right)$ coordinates and the $\left(T_{\mathrm{c}}, \Omega_{\mathrm{s}}\right)$ coordinates are systematically discussed. It indicates that the results of the usual mean-field approximation are consisted with those of the effective-field theory with correlations.

\section{ACKNOWLEDGMENT}

Project supported by the National Training Programs of Innovation and Entrepreneurship for Undergraduates (Grant No.: 201310452042).

\section{REFERENCES}

[1] R. H. Kodama, "Magnetic nanoparticles," J. Magn. Magn. Mater., vol. 200, Oct. 1999, pp. 359-372, doi: 10.1016/S0304 8853(99)00347-9

[2] O. Iglesias, A. Labarta, "Finite-size and surface effects in maghemite nanoparticles: Monte Carlo simulations," Phys. Rev. B, vol. 63, May. 2001, pp. 184416 1-11, doi: 10.1103/PhysRevB.63.184416.

[3] T. Kaneyoshi, "Phase diagrams of a nanoparticle described by the transverse Ising model," Phys. Status Solidi B, vol. 242, Oct. 2005 , pp. 2938-2948, doi: 10.1002/pssb.200540101.

[4] T. Kaneyoshi, "Magnetizations of a nanoparticle described by the transverse Ising model," J. Magn. Magn. Mater., vol. 321, Jun. 2009, pp. 3430-3435, doi: 10.1016/j.jmmm.2009.06.064.

[5] T. Kaneyoshi, "The possibility of a compensation point induced by a transverse field in transverse Ising nanoparticles with a negative core-shell coupling," Solid State Commun., vol. 152, Feb. 2012 , pp. 883-886, doi: 10.1016/j.ssc.2012.02.011

[6] T. Kaneyoshi, "Shape dependences of magnetic properties in 2D Ising nano-particles,", Phase Transitions, vol. 86, Apr. 2013, pp. 404-418, doi: 10.1080/01411594.2012.699049.

[7] Z. X. Lu, "Parameter modifications in the phase diagrams of a ferroelectric nanoparticle described by the transverse field Ising model,” Eur. Phys. J. Appl. Phys., vol. 66, Apr. 2014, pp. 10403 15, doi: 10.1051/epjap/2014130529.

[8] T. Kaneyoshi, "Transverse Ising nano-systems: Unconventinal surface effects,” J. Phys. Chem. Solids, vol. 81, Feb. 2015, pp. 6673, doi: 10.1016/j.jpcs.2015.02.004.

[9] T. Kaneyoshi, "The possibility of re-entrant phenomena induced by a transverse field in ultra-thin transverse Ising films," Phase Transitions, vol. 88, Feb. 2015, pp. 121-136, doi 10.1080/01411594.2014.961153.

[10] T. Kaneyoshi, "Unique magnetic properties of an Ising nanowire with a spin glass like disorder at the surface," Physica B, vol. 462, Apr. 2015, pp. 34-39, doi: 10.1016/j.physb.2015.01.016.

[11] T. Kaneyoshi, "Unique phase diagrams in ultra-thin Ising films with dilutions,” J. Magn. Magn. Mater., vol. 379, Apr. 2015, pp. 260-264, doi: 10.1016/j.jmmm.2014.12.046. 